\title{
Milli meter Wave MIMO-OFDMA Scheme with MMSE-based VEMF in 6G Wireless Technology
}

\author{
G. Jagga Rao ${ }^{a}$, Dr.Y. Chalapathi Rao ${ }^{b}$, Dr. Anupama Desh Pande \\ ${ }^{a}$ Research Scholar,Department of ECE, JJTU, Vidya Nagar, Rajasthan, India \\ ${ }^{\mathrm{b}}$ Associate Professor, Department of ECE, VNR VJIET, Hyderabad, Telangana, India \\ ${ }^{c}$ Professor, Department of ECE, JJTU, Vidya Nagar, Rajasthan, India \\ ag.jagadeesh458@gmail.com, ${ }^{\mathrm{b}}$ chalu.8421@gmail.com, ${ }^{\mathrm{c}}$ anupamadesh@jjtu.ac.in
}

Article History: Received: 10 November 2020; Revised 12 January 2021 Accepted: 27 January 2021; Published online: 5 April 2021

\begin{abstract}
Millimetre Wave (MmWave) massive multiple-input multiple-output (MmWave-massive-MIMO) has developed as beneficial for gigabit-per-second data broadcast into 6G digitized wireless technology. The collection of low-rate and energyefficient (EE) types of machinery, low power consumptions, multi-bit quantized massive MIMO-Orthogonal Frequency Division Multiplexing Access (OFDMA) structure have been planned for the receiver manner. The main concentration effort is the minimization of a state-of-the-art pilot-symbol quantized (PSQ) massive MIMO-OFDMA system (m-MIMO-OFDM-S). Accordingly, in this analysis, by minimizing many advantages of the Variational Estimated Message Fleeting (VEMF) algorithm. A modified low complexity manner VEMF algorithm is invented for the utilization of the ASQ-m-MIMO-OFDM-S structure. Hence, two new modules improve the energy efficiency and spectrum efficiency for wireless smart 6G technology of pilot bits allocation process for MmWave connections of the hybrid MIMO-OFDM receiver structural design. Several technologies such as massive MIMO-OFDMA, 3GPP \& 4G\& 5G technology, the device-to-device communication (D2D), GREEN communication have increasingly important consideration in assisting spectrum consumption along with power consumption during simulations. The proposed VEMF algorithm has achieved higher capacity, sum rate, Energy Efficiency (EE), and throughput for the receiver section. Finally, we present a greater number of user's data transmissions MmWavemassive-MIMO-OFDMA system.
\end{abstract}

Keywords: pilot-symbol quantized, VEMF, m-MIMO-OFDM-S, message passing

\section{Introduction}

Millimetre-wave m-MIMO-OFDM-S has turned into a valuable technology for Giga b/s information transmission in $5 \mathrm{G}$ remote correspondence as a result of its gigantic potential in improving range use, in general throughput, and vitality effectiveness [1]. Such more quantities number of antenna elements of base position bring about tremendous equipment use and vitality utilization. To accomplish minimal effort and EE hardware mechanisms, small-data quantization [2] and multi-bit quantization [3] are proposed recipient equipment design, e.g., multi-bit weight calculation m-MIMO-OFDM framework [4]. The creators explore the utilization of onesymbol AD-DC with unbounded goals bit AD-DC in an m-MIMO framework [5-6]. Our thought is to use the upsides of a best-in-class multi-symbol quantized (MSQ) m-MIMO information for the plan of a versatile bit massive MIMO design. Initially, we offer an ephemeral literature work of MSQ m-MIMO design. message to sign discovery in a PSQ m-MIMO information be not quite the same as to customary MIMO design. A high falsification of MSQ makes it hard to do exact channel state information (CSI) sufficient [7]. one more strategy for joint-channel decision and signal discovery received [8], the pilot's example with information examples towards improving the channel assessment creators structure preparation for discovery method while utilizing a lot more rapid pilot succession. The main objective variable in complex representations by minimizing adjacent symbols utilizing VE. similarly, our proposed modules can perform channel estimation, to predict and estimate all ADC power minimization at the receiver. Our activity results calculation together with MMSE-based VAMP can accomplish the higher limit, throughput rate, and EE are the most powerful communication systems.

\section{System Model}

In an existing algorithm, we had debated a 32×32 m-MIMO-OFDMA-S transmission using BPSK communication in the Rayleigh channel (RC) with MIMOCEM [21] and JAG6G [22] procedures. The simulated results with the $32 \times 32$ MIMO-OFDM system have limited specifications in wireless communication systems. In this proposed model, we discuss a different equalization approach to energy efficiency, spectrum efficiency, and power reduction also. We will reschedule that channel is a flat fading RC multipath channel and the major 
modulation is QAM/QPSK. our system model design below. In a 64×64 MIMO-OFDM channel, the possible tradition of the presented 64 transmitting antennas can be as follows:

A. Study that we have a transmission symbol for example S1, S2, S3 .....Sn.

B. In standard transmission, we will be transfer $S_{1}$ in the first-time symbol slot, $S_{2}$ in the second time symbol slot, $S_{n}$ and up to the end.

C. Yet, take 64 transmitting antennas at a time. In the primary symbols slot, send $S_{1}$ and $S_{2}$ from the primary and secondary antenna. In secondary time symbols slot send $S_{3}$ and $S_{4}$ from the primary and secondary antenna send $S_{5}$ and $S_{6}$ in the third time packet slot and up to end.

D. Now grouping 8 or 16 symbols at a time and sending them in a one-time packet slot, we need only $\frac{N}{4}$ time packet slots to the whole transmission.

E. This modest clarification of a smart m-MIMO-OFDMA-S transmission 6G technology with 64 transmit antennas and 64 receive antennas at a time.

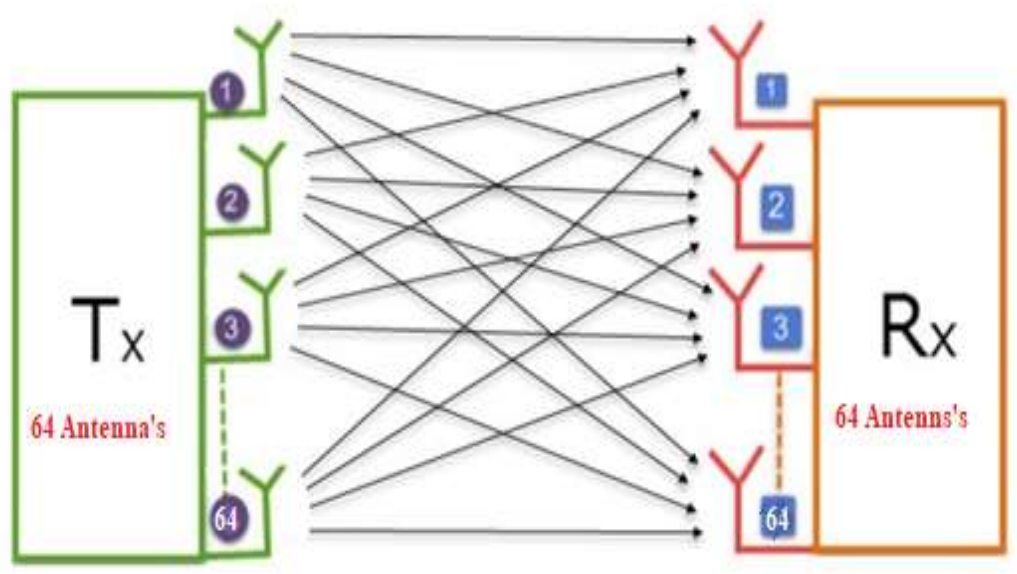

Fig. 1. Block diagram for (64 x 64) both Transmitting and receiving.

$$
\mu=\frac{1}{\sqrt{2^{\pi} \sigma^{2}}} c \frac{c n-\mu}{2 \sigma} x^{2}
$$

The main channel involvement by each transmitting antenna is the self-based station determine from the free space channel for only transmitting antenna. For transmitting pilots $i^{\text {th }}$ to receiving pilots $j^{\text {th }}$, each transmitting and receiving packet are travel free space in random data and finally $n$ has random noise is automatically added in our free space.

We study the JAGSRC [13] is a quantization model joint analysis for both transmit and receive for short-range of communication protocols determined. The rationale JAGSRC is to minimize enough ISNR, so, we have the quantized symbol's data Markov analysis equation below

$$
\mathrm{Y}=\mathrm{Q}(\mathrm{y})+\mathrm{Dy}+\mathrm{nq}
$$

In mainly to dive real and imaginary parts individually why because to remove noise data for

$\mathrm{D}=\left(1-\lambda_{0}\right)$ I number of pilot symbols corresponding in ADC's. For a speckle input distribution, correspondent in ADC s. For a random input delivery, the values of $\lambda$ for $\lambda>0.5$ can be approached by $\lambda=\frac{\pi \sqrt{3}}{2} 2^{-2 b}$. The weighted output at the $y_{g}$ receiver is

$$
y_{g}=\mathrm{Q}\left(\sqrt{g_{1 g_{2}}} h_{g}^{H} v_{g} s_{g}+n_{g}\right)
$$

$\mathrm{Q}$ is the quality of packets to transfer in free space in distributed antenna system for basic base stations

$$
\operatorname{SWR}=\sum_{i=1}^{c} h_{v}^{n} v_{t} s_{t}+\left(1-\lambda_{b}\right) n g+n q 4
$$

Therefore Signal-to-interference weighted Ratio (SWR) at the $g^{\text {th }}$ receiver and total information transit free space in the wireless communication process is

$$
\mathrm{R}=\frac{\left(1-\lambda_{b}\right) \sqrt{g_{1}} g_{2}\left|h_{g}^{H}\right|}{\lambda_{b \sqrt{g_{1} g_{2}}\left|h_{g}^{H}\right|+\sqrt{g_{1 g_{2}}}}}
$$

and we use the impartiality $\sqrt{g_{1}} g_{2}$ are gain vector data of mutually transmitter and receiver and $h_{g}^{H}$ Quantization information packet pilots for total vector, given below 


$$
\mathrm{E} \llbracket h_{g}^{H} v_{g} \rrbracket=\mathrm{E} \llbracket h_{g} \rrbracket \mathrm{E} \llbracket h_{g}^{H} v_{g} \rrbracket=\left(2^{b} \frac{F}{F-1}\right) \quad 6
$$

Next, calculate $\Delta \mathrm{R}$ as the vector-matrix CSI and defective free space channel feedback. We present estimate effects CSI- $\Delta \mathrm{R}$ difference as follows

$$
\begin{aligned}
& \Delta \mathrm{R}=£\left[\log _{2}\left(1+\frac{1-\lambda_{b} \pm \sqrt{g^{1} g 2\left|h_{g}^{H} v_{g}\right|}}{\lambda \sqrt{g^{1} g 2\left|h_{g}^{H} v_{g}\right|}+\sigma^{2}}\right)\right] \\
& \mathrm{V}^{\mathrm{E}}=\log _{2} \int_{0}^{m} \sqrt[2]{\frac{1-\lambda_{b} \pm \sqrt{g^{1} g 2\left|h_{g}^{H} v_{g}\right|}}{\left(\sqrt{g_{1 g_{2}}} \sum_{i=1}^{c} h_{v}^{h} V_{t} S_{t}+n_{q}\right.}} \quad 8
\end{aligned}
$$

The throughput of user $g_{. . . . n}$ with VE bit distribution $\mathrm{R}$ is pilot bit calculation for total matrix and optimize power with constant variable's data

$$
\text { Power }^{C}=\frac{1}{\lambda_{b}} 2^{F-1}=\frac{\sqrt[2]{3}}{3 \pi} 2^{2\left(b-\frac{B}{2(G-1)}\right)} \quad 9
$$

The VEMF algorithm deliberates both power consumption and adaptive ADC symbol allocation and it can guarantee that the Low power consumption of total hardware.

\section{Proposed Method}

In the proposed methodology to transmit a greater number of user's data transmit at a time. So, the signal indicates LW and calculates the weighted matrix. Each complex-valued are optimized and includes two real and imaginary ADCs, which perform separately dividing as fallows

$$
し \mathrm{~W}=\operatorname{sgn}(\breve{y})=\operatorname{sgn}\left(y^{R}\right)+\mathrm{I} x \operatorname{sgn}\left(y^{I}\right) \quad 10
$$

where $\mathrm{LW}$ is calculated modulation process to up to IFFT analysis for total pilot symbols with overall packets transmission in free space channel. Here QAM/QPSK modulation process transfer four bits of data transmission at a time, thus compared to existing modulation techniques.

The IFFT out weight matrix is given below

$$
\mathrm{P}(\mathrm{LW} \mid \mathrm{H}, \mathrm{X})=\pi_{f=1}^{F} \pi_{j=1}^{j} \mathrm{P}\left(y_{f j} \mid z_{f j}\right) \quad 11
$$

Where $\mathrm{P}(\mathrm{LW} \mid \mathrm{H}, \mathrm{X})$ is cyclic prefix analysis means we are adding guard intervals for total pilot symbols for all packets. here we adding only 0's (zeros).

$$
\begin{array}{ll}
\mathrm{P}\left(y_{f j} \mid z_{f j}=\mathrm{P}\left(y_{f i}^{R} \mid z_{f j}^{R}\right) \mathrm{P}\left(y_{f i}^{I} \mid z_{f j}^{I}\right)\right. & 12 \\
P_{H}\left(\mathrm{H} \mid \delta_{g}\right)=\pi_{f=1}^{F} \pi_{g=1}^{G} P_{H}\left(h_{f g} \mid \delta_{g}\right) & 13 \\
P_{H}\left(\mathrm{~h}_{f g} \mid \delta_{g}\right)=£ N\left(h_{f g} ; 0, \delta_{g}^{-1}\right) & 14
\end{array}
$$

The collective effect of transmit power $\boldsymbol{P}_{\boldsymbol{H}}$, channel fading for free-space communication analysis, and adaptive AD-DC weighted determination parameter $\boldsymbol{\delta}$ is for overall weighted matrix calculation for total FFT analysis of overall communication. Here $\mathrm{P}$ is the total pilot symbol analysis of all random input pilot bits equations (10)-(14).

$$
P_{\delta}\left(\delta_{g}\right)=\Gamma^{\prime}\left(\delta_{g} ; a_{0}, b_{0}\right)
$$

Where $\boldsymbol{P}_{\boldsymbol{\delta}}$ is the receiving pilot bits for total throughput data and $\boldsymbol{\delta}$ is a combination of original bits and random noise? so, equations (14) and (15) are analyses of receiving pilot data with a low error rate for calculating minimum mean square error data.

Algorithm steps:

Step 1: first random pilot symbols generated like no. of bits.

Step 2: Applying QAM/QPSK modulation process.

Step 3: Applying serial to parallel process which are applying our random data.

Step 4: IFFT \& FFT analysis for the transformation of all random pilot symbols. 
Step 5: Reverse process for parallel to serial communication and minimizing noise data.

Step 6: Finally calculate MMSE and throughput for total input bits.

\section{Simulation Results}

In this segment, we show the exhibition of our proposed calculation and dissect the best offloading technique for calculation offloading hybrid multiple access methods based on users' power and energy budgets. Here to optimize the improved signal to noise ratio and reduction of mean square error receiver distributed antenna system.

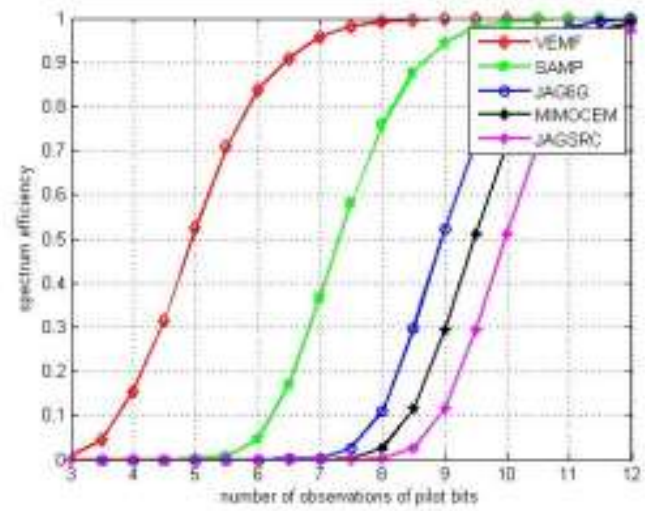

Fig 2. Spectrum Efficiency performance: throughput vs no. of base stations.

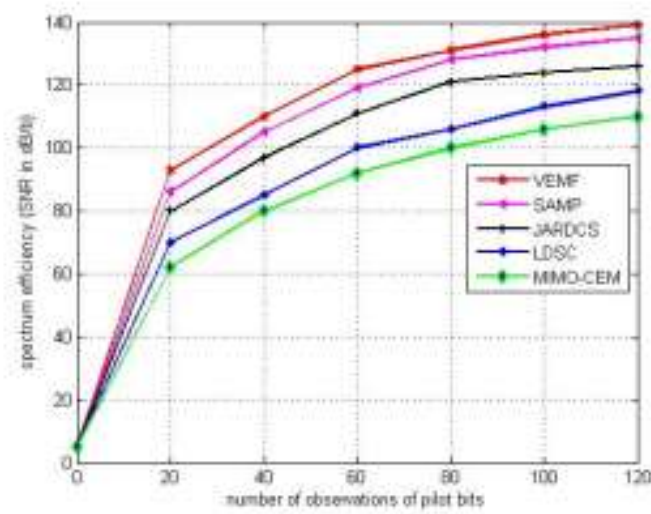

Fig 3.Spectrum Efficiency (ISNR): SNR vs number of base station users

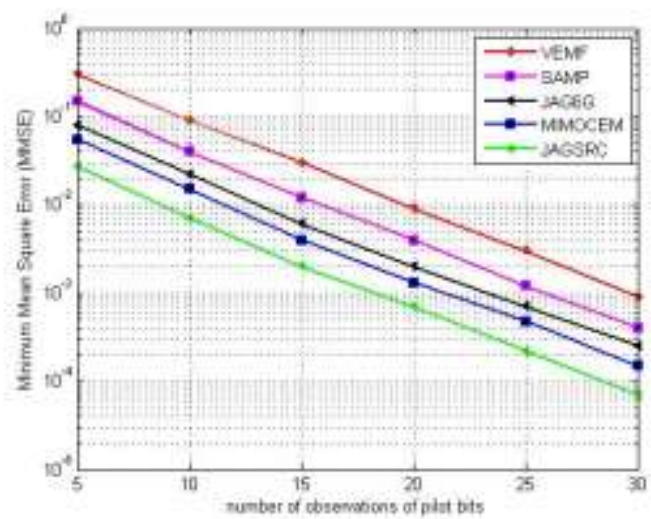

Fig 4. MMSE performance vs. no. of bits parameter initialization.

The above figure 3 describes spectrum efficiency as up to the max limit '1' in existing algorithms are below. Figures 4 and 5 are spectrum efficiency and bit error rate analysis for VEMF proposed one. 


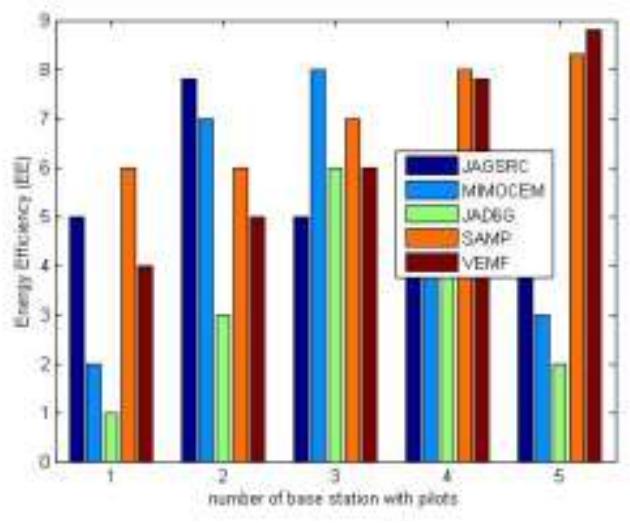

Fig 5. Number of Base stations vs. Energy Efficiency

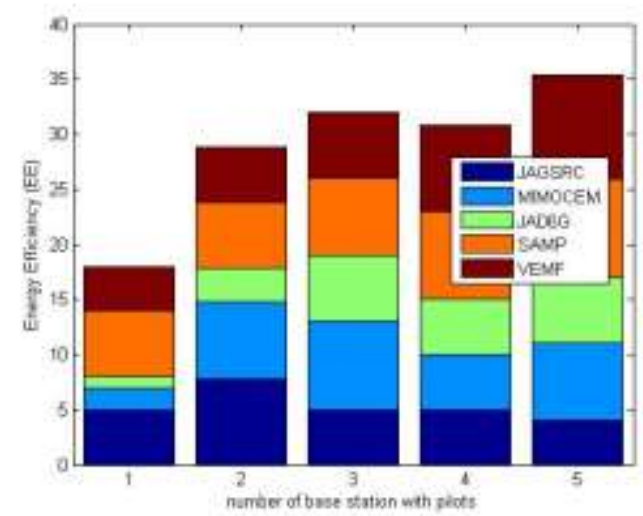

Fig 6. Several Base stations vs. Energy Efficiency with $6 \mathrm{G}$ technology.

The above figures 5 and 6 are calculated no. of base station user's vs improved signal to noise ratio. Next, we associate the presentation of four extrapolation detectors with our proposed MMSE-based VAMF, JAGSRC [20], MIMOCEM [21], JAG6G [22], SAMP [24], To choose the same simulation restrictions as used in existing works. we estimation the adaptive m-MIMO-OFDM-S with 64 antennas, 1 lakh users, and a pilot length of $J_{\text {pilot }}=64$. The outcomes show that MMSE-based VAMF could perform the channel approximation and throughput optimization with power minimization. It can attain improved concert JAGSRC and JAG6G, but MEMOCEM in high SNR. On the other hand, MMSE-based VAMF delivers well trustworthiness in assessment with MEMOCEM. When symbol pilot length is satisfactory, the performance of MMSE-based VAMF is better than JAGSRC, JAG6G but it is inferior to MEMOCEM.

\section{Conclusion}

This paper article, a novel VEMF algorithm proposed to a greater number of user's data transmission in wireless communication for $6 \mathrm{G}$ technology. Note our proposed compared four methods in existing massive MMIO systems. As we study an urban and rural deployment setup with the urban macro cell radius of $1 \mathrm{~km}$. The energetic pilot users in the communication network form spectrum efficiency and energy efficiency pairs conditional on a threshold distance criterion. In future work developed low complexity design for machine learning and deep learning with artificial intelligence system in high-quality images and live video data transmission.

\section{References}

1. M. Xiao, S. Mumtaz, Y. Huang, L. Dai, Y. Li, M. Matthaiou, G. K.Karagiannidis, E. Bjornson, K. Yang, C. L. I, and A. Ghosh, "Millimeter wave communications for future mobile networks," IEEE J. Sel. Areas Commun., vol. 35, no. 9, pp. 1909-1935, Sept 2017.

2. S. Han, I. Chih-Lin, Z. Xu, and C. Rowell, "Large-scale antenna systems with hybrid analog and digital beamforming for millimeter wave 5G," IEEE Commun. Mag., vol. 53, no. 1, pp. 186-194, Jan. 2015.

3. J. Mo and R. W. Heath, "Capacity analysis of one-bit quantized MIMO systems with transmitter channel state information,” IEEE Trans. Signal Process., vol. 63, no. 20, pp. 5498-5512, Jul. 2015. 
4. Y. Li, C. Tao, G. S. Granados, A. Mezghani, A. L. Swindlehurst, L. Liu, "Channel Estimation and Performance Analysis of One-Bit Massive MIMO Systems,” IEEE Trans. Signal Process., vol. 65, no. 15, pp. 4075 - 4089, Aug. 2017.

5. N. Liang and W. Zhang, "Mixed-ADC massive MIMO," IEEE J. Sel. Areas Commun., vol. 34, no. 4, pp. 983997, Mar. 2016.

6. T. C. Zhang, C.-K.Wen, S. Jin, and T. Jiang, "Mixed-ADC massive MIMO detectors: Performance analysis and design optimization," IEEE Trans. Wireless Commun., vol. 15, no. 11, pp. 7738-7752, Nov. 2016.

7. C. K. Wen, C. J. Wang, S. Jin, and K. K. Wong, "Bayes-Optimal Joint Channel-and-Data Estimation for Massive MIMO With Low-Precision ADCs,” IEEE Trans. Signal Process., vol. 64, no. 10, pp. 2541-2556, 2015.

8. V. Buchoux, O. Capp'e, ' E. Moulines, and A. Gorokhov, "On the performance of semi-blind subspace-based channel estimation," IEEE Trans. Signal Process., vol. 48, no. 6, pp. 1750-1759, 2000. [9] M. Bayati and A. Montanari, "The dynamics of message passing on dense graphs, with applications to compressed sensing," IEEE Trans. Inform. Theory, vol. 57, no. 2, pp. 764-785, 2011.

9. D. G. Tzikas, A. C. Likas, and N. P. Galatsanos, “The variational approximation for bayesian inference," IEEE Trans. Signal Process., vol. 25, no. 6, pp. 131-146, 2008.

10. J. T. Parker, P. Schniter, and V. Cevher, "Bilinear Generalized Approximate Message Passing -Part II: Applications," IEEE Trans. Signal Process., vol. 62, no. 22, pp. 5854-5867, 2014.

11. Z. Zhang, X. Cai, C. Li, C. Zhong, and H. Dai "One-bit Quantized Massive MIMO Detection Based on Variational Approximate Message Passing,” IEEE Trans. Signal Process., vol. 66, no. 9, pp. 2358-2373, 2018.

12. O. Orhan, E. Erkip, and S. Rangan, "Low power analog to digital conversion in millimeter wave systems: Impact of resolution and bandwidth on performance," in Proc. IEEE Inf. Theory Appl. Workshop, Feb. 2015, pp. 191-198.

13. N. Jindal, "MIMO broadcast channels with finite-rate feedback," IEEE Trans. Inf. Theory, vol. 52, no. 11, pp. 5045-5060, Nov 2006.

14. De Carvalho and D. Slock, "Semi-blind maximum-likelihood multichannel estimation with gaussian prior for the symbols using soft decisions," in IEEE Veh. Technol. Conf. (IEEE VTC), vol. 2, 1998, pp. 1563-1567

15. G. Tzikas, A. C. Likas, and N. P. Galatsanos, “The Variational Approximation for Bayesian Inference,” IEEE Trans. Signal Process., vol. 25, no. 6, pp. 131-146, 2008.

S. Rangan, J. T. Parker, P. Schniter, and etc., "Gampmatlab," https:// sourceforge.net/projects/gampmatlab/.

16. Risi, D. Persson, and E. G. Larsson, XMassive MIMO with 1-bit ADC,s ArXiv e-prints, arXiv:1404.7736, Apr. 2014. https://arxiv.org/pdf/1404.7736.

17. G. Jagga Rao " ECG de-noising by using jag-wavelet filter for Heartbeat noise signals" in Volume 4 Issue 4 , pp. 220-225, April 2018.

18. G. Jagga Rao, Y. Chalapathi Rao " Robust Analysis of Minimizing PAPR Reduction by Using Low Density Symbol Check "in Volume 3 Issue 5, pp. 1104-1109, June 2018.

19. G. Jagga Rao, Y. Chalapathi Rao " Robust Bit Error Rate Optimization for MASSIVE MIMOCEM System using Channel Coding Method "in Volume 8-Issue 4S2, pp. 180-184, March 2019.

20. G. Jagga Rao, Y. Chalapathi Rao " Artificial Intelligence \& Machine Learning Based Wireless MIMOOFDM Communication system in JAG6G Analysis "in Volume 8-Issue 4, pp. 3740-3755, May2019.

21. G. Jagga Rao, Y. Chalapathi Rao, Dr. Anupama Desh Pande "A Novel Approach for High Secured Image Transmission in 6G via MIMO-OFDMA process in NCHAOS Encryption Algorithm" in Volume 9-Issue 10, pp. 1481-1492, Oct 2019.

22. G. Jagga Rao, Y. Chalapathi Rao, Dr. Anupama Desh Pande "Detection For 6G-NOMA Based Machine Learning Optimization for Successive Adaptive Matching Pursuit Analysis", Q3, pp. 1803-1812, Jan 2020.

Sudha, Y. Chalapathi Rao, G. Jagga Rao " Machine Learning based Copy-Move Forgery Detection with Forensic Psychology Ultra-Hd images "in Volume 81, Nov-Dec-2019. 


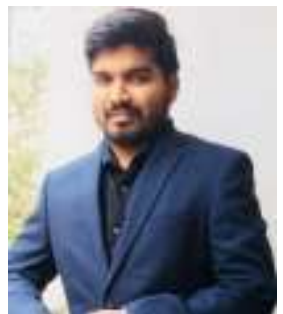

G. Jagga Rao received the $\mathrm{B}$. Tech degree in Electronics and Communication Engineering from the Jawaharlal Nehru Technological University, Kakinada of Andhra Pradesh in 2013, the MTech. degree in communication systems from the Andhra University of Andhra Pradesh in 2015, and the pursuing Ph.D. degree in Electronics and Communication Engineering from the University of Shri jagdishprasad jhabarmal tibrewala University. He is currently a Research in the Department of Electronics and Communication Engineering at the vincense software solutions Pvt. ltd. His research interests include wireless sensor cooperative networks, mobile communication for antennas, Deep learning Machine learning, Artificial Intelligent, convolution neural networks, Digital Image Processing, Digital signal Processing and gesture applications in smart devices at speech reorganization systems.

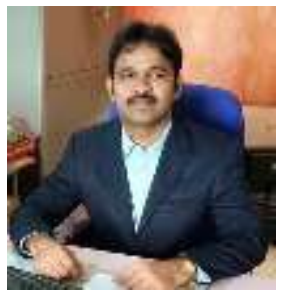

Dr. Y. Chalapathi Rao received the B. Tech degree in Electronics and Communication Engineering from the ANU University Guntur of Andhra Pradesh in 2005, the M. Tech. degree in communication systems from the JNTUK University of Andhra Pradesh in 2009, and the Ph.D. degree in Electronics and Communication Engineering from the University Nagarjuna University. His research interests include wireless sensor cooperative networks, mobile communication for antennas, Deep learning, Machine learning, Artificial Intelligent, convolution neural networks, Digital Image Processing, Digital signal Processing and gesture applications in smart devices at VLSI system design.

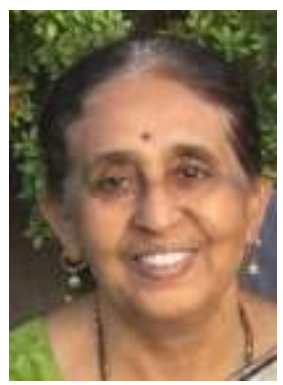

Dr. Anupama Desh Pande working in Electronics and Communication Engineering department from the JJTU university. Her research interests include wireless sensor cooperative networks, mobile communication for antennas, Deep leaning, Machine learning, Artificial Intelligent, convolution neural networks 